\title{
Differentially expressed genes between primary cancer and paired lymph node metastases predict clinical outcome of node-positive breast cancer patients
}

\author{
Yumei Feng • Baocun Sun · Xiaoqing Li · Liang Zhang · Yun Niu • \\ Chunhua Xiao - Liansheng Ning · Zhiyi Fang - Yuli Wang · Lina Zhang • \\ Jing Cheng $\cdot$ Wei Zhang $\cdot$ Xishan Hao
}

Published online: 15 February 2007

(C) Springer Science+Business Media B.V. 2007

\section{Erratum to: Breast Cancer Res Treat DOI 10.1007/s10549-006-9385-7}

Unfortunately a wrong table was printed in this article. Please find below the correct Table 3 .

As a result the text also contains incorrect information. Please find below the correct information:

Identification of Candidate Differentially Expressed Genes

Seventy-nine differentially expressed genes between primary cancers and paired lymph node metastases were identified (Table 3), consisting of 50 genes downregulated and 29 up-regulated. The functions of these genes were related to cell migration and adhesion, extracellular matrix, cell growth, signal transduction, and metabolism, which are involved in biology process of metastasis.

The online version of the original article can be found at http:// doi.org/10.1007/s10549-006-9385-7

Y. Feng $\cdot$ B. Sun $\cdot$ X. Li · Y. Niu · C. Xiao .

L. Ning $\cdot$ Z. Fang $\cdot$ Y. Wang $\cdot$ L. Zhang .

X. Hao $(\bowtie)$

Breast Cancer Prevention and Treatment Key Laboratory of Ministry of Education, Tianjin Medical University Cancer

Institute and Hospital, Tianjin 300060, China

e-mail: ymfeng@tijmu.edu.cn

L. Zhang $\cdot$ J. Cheng

National Engineering Research Center for Beijing Biochip

Technology, Beijing, China

\section{W. Zhang}

Department of Pathology, Cancer Genomics Core

Laboratory, The University of Texas MD Anderson Cancer

Center, Houston, TX, USA 


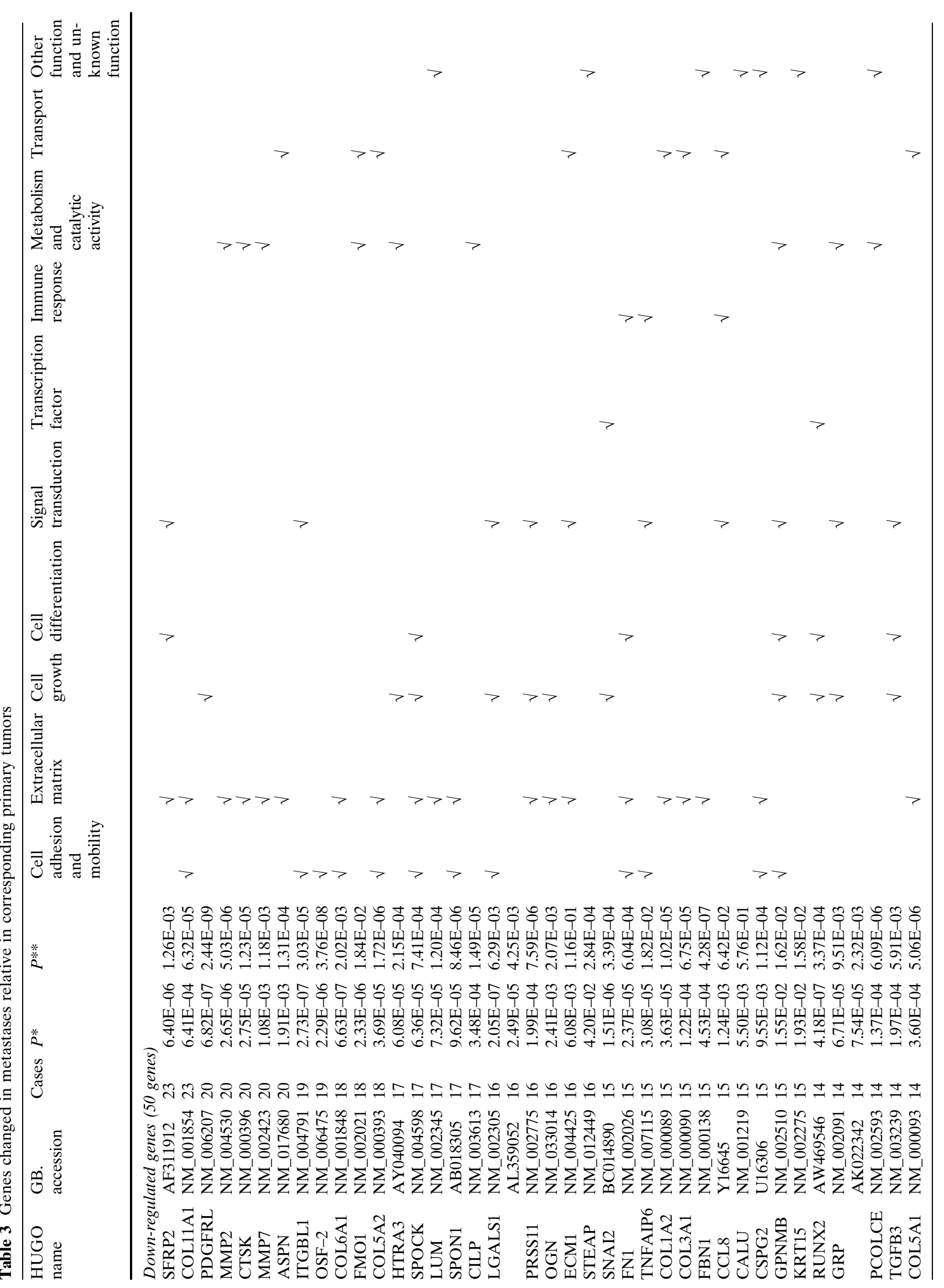




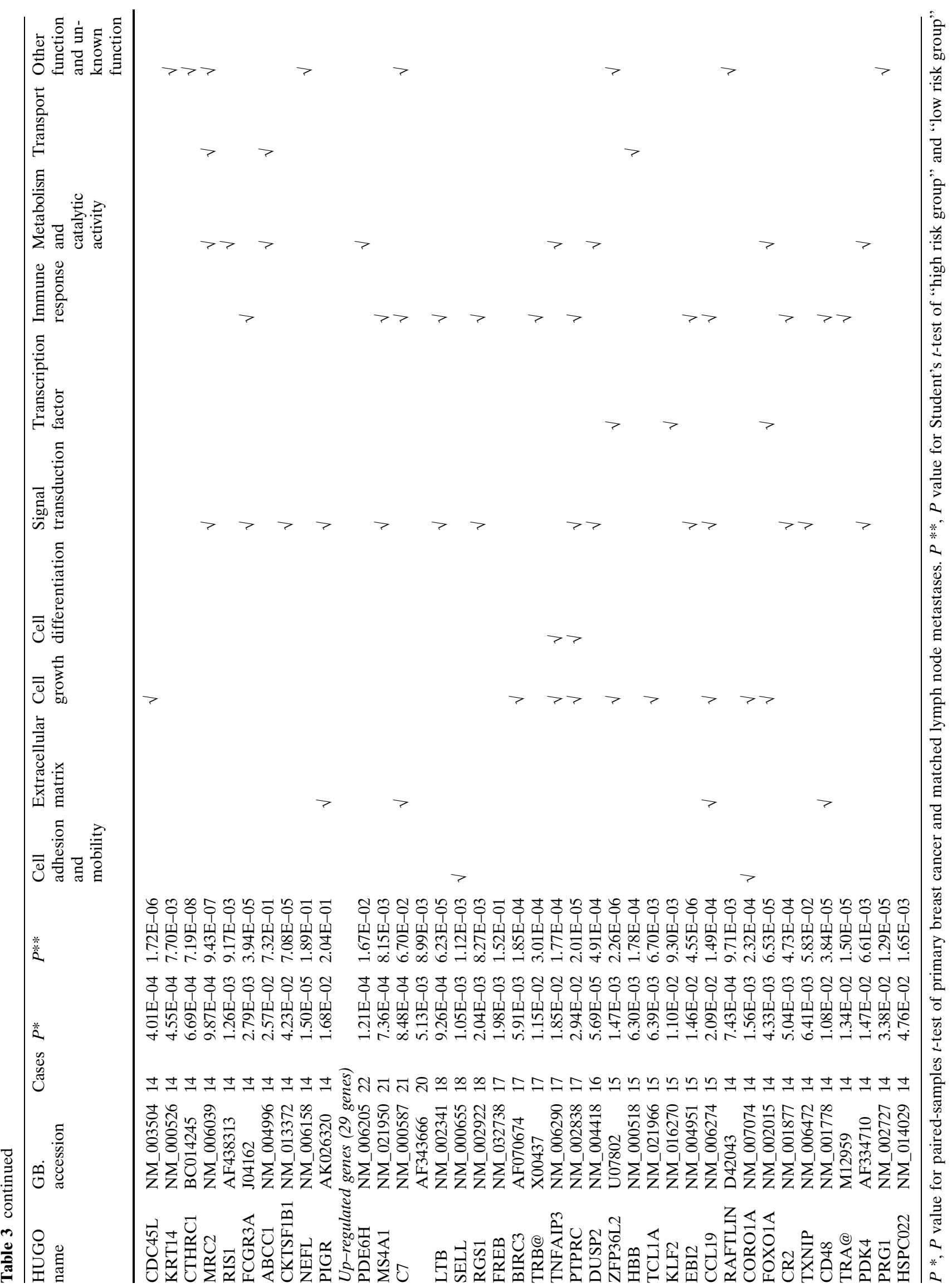

